PART 3.

Outflows, Shocks, PDRs and Masers 
Y. C. Minh and E. F. van Dishoeck, eds.

\title{
Observations of Molecular Hydrogen in Shocks and PDRs with the Infrared Space Observatory
}

\author{
Christopher M. Wright \\ School of Physics, University College, Australian Defence Force \\ Academy, University of New South Wales, Canberra ACT 2600, \\ Australia
}

\begin{abstract}
A review is presented of ISO observations of molecular hydrogen and its isotopic species, HD, toward various Galactic source types, such as shocks (Orion, DR 21, Cep A), photon dominated regions (IC $1396 \mathrm{~A}, \mathrm{~S} 140$, Orion Bar) and putative X-ray excited sources (RCW 103, SS 433). In so doing I examine the similarities and differences in the $\mathrm{H}_{2}$ spectrum found under these different excitation conditions and mechanisms, and how the observations impact on some of the latest shock and PDR models. For instance, in addition to the ubiquitous $\sim 2000 \mathrm{~K}$ component in shocks, seen using ground-based instrumentation, ISO reveals the existence of gas with both lower $(150-800 \mathrm{~K})$ and higher $(\geq 3000 \mathrm{~K})$ excitation temperatures. Further, ISO allows for the first time a measure of the bulk gas temperature toward PDRs $(100-700 \mathrm{~K})$, and $\mathrm{H}_{2}$ is also detected where X-ray emission is observed, providing at least circumstantial evidence of X-ray heating.
\end{abstract}

\section{Introduction}

Before the launch of the Infrared Space Observatory (ISO, Kessler et al. 1996) observations of molecular hydrogen, $\mathrm{H}_{2}$, were restricted to a hot component with an excitation temperature of about $2000 \mathrm{~K}$ in shock excited sources, and a nonthermally (i.e. fluorescently) excited component in photon dominated regions (PDRs). These components were typically probed with the 1-0 S(1) line at 2.12 $\mu \mathrm{m}$ as well as several other near-infrared ro-vibrational transitions, and in some cases pure rotational transitions from high $J$ levels (e.g. Gredel 1994; Oliva \& Moorwood 1988; Knacke \& Young 1981). Only a handful of observations, principally toward the Orion star-forming region (Genzel \& Stutzki 1989), of lower energy pure rotational transitions existed, e.g. the $0-0 \mathrm{~S}(2)$ and $0-0 \mathrm{~S}(1)$ lines at 12.2786 and $17.0348 \mu \mathrm{m}$, but which already pointed to the existence of a lower temperature component in such sources (e.g. Beck, Lacy, \& Geballe 1979; Parmar, Lacy, \& Achtermann 1991, 1994; Richter et al. 1995; Burton \& Haas 1997).

From the point of view of star formation studies, the over-riding motivating factor behind ISO $\mathrm{H}_{2}$ observations is simply that in molecular clouds, where stars are forming, most of the mass resides in $\mathrm{H}_{2}$. Further, emission from the lowest pure rotational transitions, where most of the $\mathrm{H}_{2}$ mass is expected to reside, 
occurs in the mid-infrared, i.e. $5-30 \mu \mathrm{m}$, to which the Earth's atmosphere is opaque. Therefore, by opening up the window beyond $2.4 \mu \mathrm{m}$, ISO offered the possibility of a much stronger observational effort to be directed towards $\mathrm{H}_{2}$ spectroscopy of Galactic shocks, PDRs and X-ray dominated regions, XDRs, as well as external galaxies and the solar system. For example, whilst it is obvious that observing the bulk of the mass of the bulk gas constituent must lead to new knowledge, some of the more specific issues which could be addressed by such observations, include:

- How much gas exists at both higher and lower temperatures than $2000 \mathrm{~K}$ in shocks?

- What contribution does $\mathrm{H}_{2}$ make toward the total cooling of the gas behind shocks?

- What is the temperature structure of the gas in PDRs?

- What is the $\mathrm{H}_{2}$ ortho-para abundance ratio?

- When ISO observations confront theoretical models of shocks and PDRs, how do the models fare?

- Is there a unique $\mathrm{H}_{2}$ signature of $\mathrm{X}$-ray heated gas?

- How do Galactic observations impact on the interpretation of $\mathrm{H}_{2}$ data of other galaxies?

- What is the $\mathrm{HD} / \mathrm{H}_{2}$ ratio, and thereby deuterium abundance, in molecular clouds?

In the following I will review the ISO observations of molecular hydrogen and its isotopic species, HD, toward various Galactic source types, such as shocks (e.g. Orion, DR 21, Cep A), photon dominated regions (e.g. IC 1396A, S140, Orion Bar) and putative X-ray excited sources (e.g. RCW 103, SS 433). In so doing I will examine the similarities and differences in the $\mathrm{H}_{2}$ spectrum found under these different excitation conditions and mechanisms, and how the observations impact on some of the questions enumerated above. I will also briefly discuss observations of external galaxies, and the contribution of ISO to the field of 'deuteronomy'.

\section{Properties of the $\mathbf{H}_{2}$ Molecule}

Molecular hydrogen is a homonuclear linear molecule, which comprises the bulk of the molecular material in star-forming molecular clouds. Gas phase reactions are predicted to be too slow in producing $\mathrm{H}_{2}$, such that most of it is thought to be produced via surface reactions on dust grains (Hollenbach \& Salpeter 1971). Being homonuclear, $\mathrm{H}_{2}$ possesses no dipole moment, so that all radiative transitions are quadrupolar, where the angular momentum quantum number, $J$, changes by \pm 2 , whilst the vibrational quantum number, $v$, selection rule is $|\Delta v| \geq 0$. It exists in two (almost independent) states, namely ortho- $\mathrm{H}_{2}$ and para- $\mathrm{H}_{2}$. The former occurs when the spins of the two spin $\frac{1}{2} \mathrm{H}$ nuclei are 
parallel, whilst para- $\mathrm{H}_{2}$ occurs when the spins are antiparallel. All odd $J$ states are ortho, whilst all even $J$ states are para. There are clearly no radiative transitions between ortho- and para- $\mathrm{H}_{2}$, whilst in a fully molecular gas there are also no collisional transitions. Ortho-para exchange reactions may however occur in collisions between $\mathrm{H}_{2}$ and, for example, $\mathrm{H}$ and $\mathrm{H}^{+}$. In thermodynamic equilibrium above about $300 \mathrm{~K}$ the ortho-para abundance ratio is equal to the ratio of their statistical weights, i.e. 3 , whilst for lower temperatures the $\mathrm{o} / \mathrm{p}$ ratio is $<3$ (e.g. Burton, Hollenbach, \& Tielens 1992). The lowest pure rotational transition of $\mathrm{H}_{2}$ occurs at $28.2188 \mu \mathrm{m}$, the $0-0 \mathrm{~S}(0)$, which arises from $J=2$ at $510 \mathrm{~K}$ above ground. The next lowest is at $17.0348 \mu \mathrm{m}$, the $0-0 \mathrm{~S}(1)$, from $J=3$ at $1015 \mathrm{~K}$. A thorough review of $\mathrm{H}_{2}$ is given by Shull \& Beckwith (1982).

\section{Shocks}

Very briefly, a shock occurs when a wind or outflow from a star hits the ambient medium, i.e. a molecular cloud, imparting its mechanical energy and thereby heating the gas. The $\mathrm{H}_{2}$ is excited by collisions with other $\mathrm{H}_{2}$ molecules or with $\mathrm{H}$ atoms, the two primary colliding partners. Shocks occur as two broad types, continuous (or C-type), where the shock is mediated by an ambient magnetic field and the flow parameters such as velocity, temperature and density, change smoothly across the shock front; and jump (or J-type) where they change discontinuously across the front. A thorough review of shock physics is given by Draine \& McKee (1993).

The first infrared detection of shocked molecular hydrogen was made toward the Orion BN/KL cluster of massive YSOs by Gautier et al. (1976), in the $v=1-0, J=3-1$, or $1-0 \mathrm{~S}(1)$, transition at $2.12 \mu \mathrm{m}$. Since then many other transitions have been discovered, but still principally confined to the near-infrared spectral region, where the upper levels are typically at $\geq 6000 \mathrm{~K}$ above ground. These transitions are mainly sensitive to gas at a temperature of $\sim 2000 \pm 500 \mathrm{~K}$. However, based on energy arguments, it was thought to be highly unlikely that the bulk of the gas could be at such a high temperature. Indeed, pioneering observations by Beck, Lacy, \& Geballe (1979), Parmar, Lacy, \& Achtermann (1994) and Burton \& Haas (1997) toward Orion, and Richter et al. (1995) toward IC 443, of the $0-0 \mathrm{~S}(1)$ and $0-0 \mathrm{~S}(2)$ lines at 17.0 and $12.3 \mu \mathrm{m}$ respectively (upper levels at 1015 and $1680 \mathrm{~K}$ ) hinted that this was the case. However, with only two lines observed toward only 2 (perhaps atypical) sources, the statistics were clearly quite bare. ISO has changed that situation dramatically, and Table 1 presents a summary of most of those $\mathrm{H}_{2}$ observations that have been published (or are soon to be published) since ISO's launch in November 1995.

In Table 1 the excitation temperature, $T_{e x}$, has been calculated in the ground vibrational state between upper levels of $J=3(\sim 1000 \mathrm{~K}$ above ground) and 7 ( $\sim 4600 \mathrm{~K}$ above ground), i.e. using the $0-0 \mathrm{~S}(1)$ through $\mathrm{S}(5)$ transitions, and the column density of this warm gas, $N_{\mathrm{H}_{2}}$, has been calculated assuming the kinetic temperature is equal to $T_{e x}$. All transitions are typically observed in this range, and in a population diagram (Goldsmith \& Langer 1999) the data defines a relatively straight line. One can see that, apart from $\mathrm{T}$ Tau, $T_{e x}$ has quite a narrow range, from $\sim 600$ to $800 \mathrm{~K}$. Comparison with the C-type planar shock models of Kaufman \& Neufeld (1996, KN96) usually requires at least two com- 
Table 1. List of published shock sources observed by ISO.

\begin{tabular}{lccccccccc}
\hline Source & $\begin{array}{c}T_{e x}{ }^{a} \\
\mathrm{~K}\end{array}$ & $\begin{array}{c}\mathrm{N}_{\mathrm{H}_{2}{ }^{b}} \times 10^{20} \mathrm{~cm}^{-2} \\
\mathrm{~km} \mathrm{~s}^{-1}\end{array}$ & $\begin{array}{c}v_{s 1} \\
\mathrm{~cm}^{-3}\end{array}$ & $\Phi_{s 1}$ & $\begin{array}{c}v_{s 2} \\
\mathrm{~km} \mathrm{~s}^{-1}\end{array}$ & $\begin{array}{c}n_{s 2} \\
\mathrm{~cm}^{-3}\end{array}$ & $\Phi_{s 2}$ & Ref. $^{c}$ \\
\hline Cep A West & 688 & 1.8 & 20 & $10^{4}$ & 0.5 & 35 & $10^{6.5}$ & 0.001 & 1 \\
Cep A East & 740 & 1.2 & 20 & $10^{6}$ & & & & & 2 \\
DR 21 West & 628 & 5.8 & 20 & $10^{4}$ & 1.25 & 30 & $10^{6}$ & 0.005 & 3 \\
DR 21 East & 671 & 4.4 & 20 & $10^{4}$ & 1.25 & 30 & $10^{6}$ & 0.0055 & 3 \\
Orion IRc2 & 698 & 12 & 20 & $10^{4}$ & 3.0 & 35 & $10^{6.5}$ & 0.0175 & 4,5 \\
Orion Pk2 & 819 & 11 & 20 & $10^{4}$ & 4.0 & 35 & $10^{6.5}$ & 0.03 & 5 \\
Orion Pk1 & 767 & 16 & & & & & & & 6 \\
IC 443 & 822 & 2.1 & 20 & $10^{4}$ & 0.85 & 40 & $10^{6}$ & 0.005 & 7 \\
T Tau & 440 & 0.15 & & & & & & & 8 \\
HH 54 & 650 & 0.9 & & & & & & & 9 \\
\hline
\end{tabular}

${ }^{a}$ Excitation temperature, $T_{e x}$, calculated between the extinction corrected $v=0, J=3$ and 7 levels.

${ }^{b}$ Column density, $N_{\mathrm{H}_{2}}$, calculated assuming the kinetic temperature is equal to $T_{e x}$.

${ }^{c}$ (1) Wright et al. (1996), (2) van den Ancker et al. (1998), (3) Wright, Timmermann \& Drapatz (1997), (4) van Dishoeck et al. (1998), (5) Wright et al. (in preparation), (6) Rosenthal et al. (1999), (7) Cesarsky et al. (1999), (8) van den Ancker et al. (1999), (9) Neufeld, Melnick, \& Harwit (1998)

ponents to adequately match the data. These are also indicated in Table 1 . The first component is usually of a low density, low velocity and high covering factor, which reproduces the warm, $\sim 700 \mathrm{~K}$ gas, whilst the second component is usually of a higher density, higher velocity and low covering factor, and reproduces the hot, $\sim 2000 \mathrm{~K}$ gas observed with both ISO and ground-based instruments. Whilst the low density of the first component usually provides the best match with the data, the gas temperature within shocks is not much dependent on the density, as shown by van den Ancker et al. (1998), so that reasonable matches with the data may be obtained with only a single density; for example, for Orion $\mathrm{Pk} 2$ a density of $10^{6.5} \mathrm{~cm}^{-3}$ but with a lower covering factor for the first shock component works reasonably well. It is however not clear that the success at matching these shocks with the KN96 models provides unequivocal support for the models, since Rosenthal et al. (in preparation) have shown that a range of shock models in the literature can adequately match the $\mathrm{H}_{2}$ data toward the $\mathrm{Pk}$ 1 position of the Orion shock.

Perhaps the most striking feature of Table 1 is how broadly similar all the sources are. So far there has been only one identification of a J-type shock, that of T Tau by van den Ancker et al. (1999), whilst new time dependent shock models of Flower \& Pineau des Forêts (1999) have been applied to ISOCAM observations of the supernova remnant IC 443 by Cesarsky et al. (1999).

\subsection{Orion}

An example of a $\mathrm{H}_{2}$ ro-vibrational spectrum toward the Orion shock is shown in the left panel of Figure 1, whilst the extinction corrected population diagram, together with the predictions of a two-component KN96 shock model, is shown in the right panel. The precise pointing for Figure 1 was toward IRc2, where $T_{e x}$ is about $100 \mathrm{~K}$ less than toward the $\mathrm{Pk} 2$ position (defined by Beckwith et al. 1978). The $28.2 \mu \mathrm{m} 0-0 \mathrm{~S}(0)$ line was not detected toward IRc2, due to 

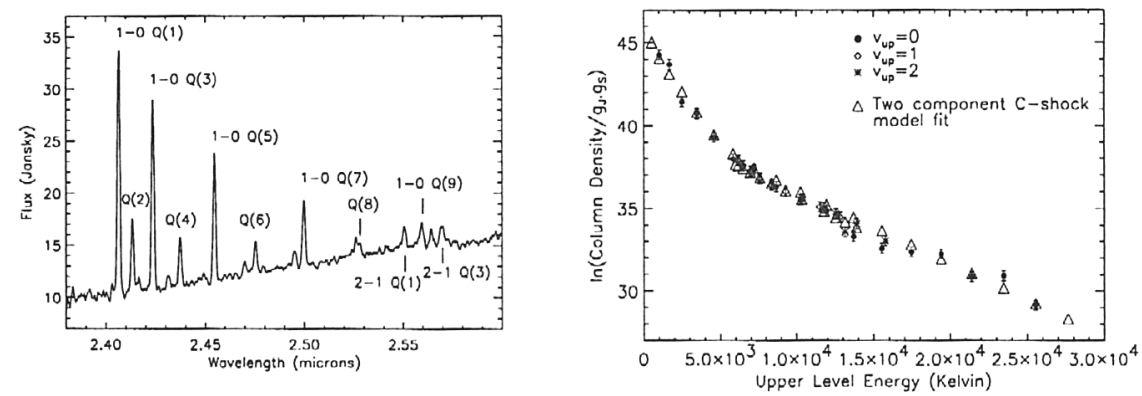

Figure 1. A segment of the $\mathrm{H}_{2}$ ro-vibrational spectrum toward the Orion shock, and the resulting extinction corrected population diagram. All $\mathrm{H}_{2}$ lines are marked, whilst all other emission lines are due to hydrogen recombination transitions from $n \rightarrow 5$.

the extremely high continuum emission of $\sim 40000 \mathrm{Jy}$, but a possible detection was made toward $\mathrm{Pk} 2$ where the continuum is weaker, $\sim 10000 \mathrm{Jy}$. In that case the excitation temperature between the $J=2$ and $J=3$ levels is $\sim 145 \mathrm{~K}$ and the column density of this cool component is of order $7 \times 10^{22} \mathrm{~cm}^{-2}$. A two-component KN96 shock model can adequately account for the warm $\mathrm{H}_{2}$, but does not reproduce the cool gas. It may be that yet another component, of a lower velocity, e.g. $5-10 \mathrm{~km} \mathrm{~s}^{-1}$, is required. Alternatively, some of the cool and even warm $\mathrm{H}_{2}$ emission may be arising from the PDR at the cloud surface, rather than from the shock, since the $\mathrm{H}_{2}$ column density there is $(1-3) \times 10^{22}$ $\mathrm{cm}^{-2}$ (Genzel \& Stutzki 1989). Toward the $\mathrm{Pk} 2$ and $\mathrm{Pk} 1$ positions even more $\mathrm{H}_{2}$ lines are observed than at IRc2 (e.g. Wright et al., in preparation; Rosenthal et al. 1999), specifically high $J$ pure rotational lines at upper levels of $\geq 25000 \mathrm{~K}$, which imply the existence of gas hotter than $2000 \mathrm{~K}$, i.e. excitation temperatures $\geq 3000 \mathrm{~K}$. This also cannot be reproduced by a two-component KN96 shock model, and Bertoldi et al. (1999a) suggest instead that high velocity, inelastic $\mathrm{H}_{2}$-ion collisions, or formation pumping, may be responsible.

\section{Photon Dominated Regions}

Photon Dominated Regions, or PDRs, occur at the interface between fully ionised $\mathrm{H}$ II regions and cold molecular clouds, where ultraviolet radiation incapable of ionising atomic hydrogen excites $\mathrm{H}_{2}$ into upper electronic states. These then decay via a cascade through the ground vibrational states. The $\mathrm{H}_{2}$ is therefore excited by fluorescence, apart from the lowest several pure rotational states (e.g. $J<10$ ) which are still collisionally excited by primarily $\mathrm{H}$ and $\mathrm{H}_{2}$. The ultimate heating of the gas is dominated by photoelectrons from dust grains and $\mathrm{H}_{2}$ vibrational heating. A review of PDRs is given by Hollenbach \& Tielens (1997).

The first infrared detection of fluorescently excited molecular hydrogen was made toward the planetary nebula NGC 7027 by Treffers et al. (1976). However, due to the nature of the excitation mechanism, such observations did not provide any information on the temperature of the gas. For these sources, only obser- 
vations of the lower pure rotational transitions could provide such information. Before ISO, only one such observation had been made, those of Parmar, Lacy, \& Achtermann (1991) toward the Orion Bar. They found a temperature gradient from $1000 \mathrm{~K}$ down to $400 \mathrm{~K}$, decreasing with increasing depth into the PDR. As for shocks, since ISO's launch the situation has changed dramatically, and Table 2 presents most of those observations of PDRs that have been published, or are soon to be published. For all the sources in Table 2 the gas density is of order $10^{5}-10^{6} \mathrm{~cm}^{-3}$, whilst the radiation field ranges from $10^{3}-10^{5} \mathrm{G}_{0}$, where $\mathrm{G}_{0}$ represents the standard interstellar radiation field (e.g. Burton, Hollenbach, \& Tielens 1992). Apart from the H2 and PDR positions in S106, the excitation temperature and column density are defined in an analogous manner to those for shocks considered above. In the case of the two $\mathrm{S} 106$ positions, $\mathrm{T}_{e x}$ is calculated between upper $J$ levels of 4 and 7, i.e. using the $0-0 \mathrm{~S}(2)$ through $\mathrm{S}(5)$ lines, since the $\mathrm{S}(1)$ through $\mathrm{S}(5)$ transitions did not fall on a straight line in a population diagram. The excitation temperature between upper $J$ levels of 3 and 4 for S106 $\mathrm{H} 2$ is $380 \mathrm{~K}$, whilst for S106 PDR it is $303 \mathrm{~K}$.

Table 2. Observed properties of PDRs.

\begin{tabular}{|c|c|c|c|}
\hline Object & $\begin{array}{l}\mathrm{T}_{e x}{ }^{a} \\
(\mathrm{~K})\end{array}$ & $\begin{array}{c}\mathrm{N}_{\mathrm{H}_{2}}{ }^{b} \\
\left(\times 10^{20} \mathrm{~cm}^{-2}\right)\end{array}$ & Ref. $^{c}$ \\
\hline S140 & 500 & 2.1 & 1 \\
\hline IC $1396 \mathrm{~A}$ pos 1 & 412 & 0.3 & 2 \\
\hline IC $1396 \mathrm{~A}$ pos 2 & 448 & 0.8 & 2 \\
\hline $\mathrm{S} 106 \mathrm{H} 2$ & 705 & 0.8 & 2 \\
\hline S106 PDR & 778 & 0.4 & 2 \\
\hline S106 IRS4 & 490 & 9.1 & 3 \\
\hline Orion Bar & 552 & 7.1 & 2 \\
\hline NGC 7023 & 580 & 0.9 & 4 \\
\hline IC 63 & 685 & 0.1 & 5 \\
\hline
\end{tabular}

${ }^{a}$ Excitation temperature, $T_{e x}$, calculated between the extinction corrected $v=0, J=3$ and 7 levels, apart from S106 H2 and S106 PDR (see text).

${ }^{b}$ Column density, $N_{\mathrm{H}_{2}}$, calculated assuming the kinetic temperature is equal to $T_{e x}$.

c(1) Timmermann et al. (1996), (2) Wright et al. (in preparation), (3) van den Ancker et al. (1998), (4) Bertoldi et al. (in preparation), (5) Thi et al. (1999)

One can see from Table 2 that there is quite a large range in $T_{e x}$, from $\sim 400 \mathrm{~K}$ up to $700 \mathrm{~K}$ and even $800 \mathrm{~K}$. As noted by Draine \& Bertoldi (1999) such high temperatures were unexpected based on pre-ISO PDR models, and suggested that perhaps a higher photoelectric heating efficiency was required. Indeed, Weingartner \& Draine (1999) have calculated a new efficiency, based on an enhanced dust-to-gas ratio in the PDR due to gas-grain drift, and there is now good agreement between the models and data (e.g. Draine 2000). In those sources where the $28.2 \mu \mathrm{m} 0-0 \mathrm{~S}(0)$ line was detected, the excitation temperature between the $J=2$ and 3 levels ranges from $\sim 100 \mathrm{~K}$ (IC 63) to $170 \mathrm{~K}$ (IC 1396A Pos 2, Orion Bar), with at least an order of magnitude greater column density 

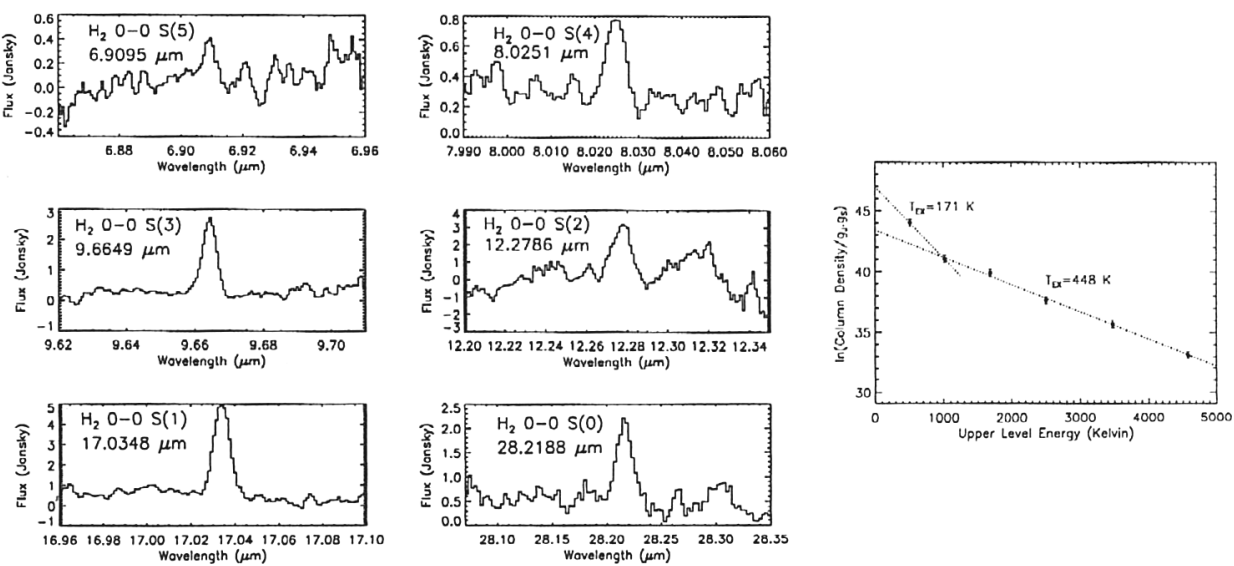

Figure 2. Spectra of several $\mathrm{H}_{2}$ pure rotational lines observed toward a position in the reflection nebula IC 1396A, and the resulting extinction corrected population diagram.

in this cool $\mathrm{H}_{2}$. There is also an apparently much hotter gas component present, revealed through detection of high $J$ lines, e.g. $0-0 \mathrm{~S}(15)$ from $J=17$ at 21400 $\mathrm{K}$ in the Orion Bar, and $0-0 \mathrm{~S}(11)$ from $J=13$ at $13700 \mathrm{~K}$ in S106, but which Draine \& Bertoldi (1999) suggest may arise from ultraviolet and/or formation pumping. In the case of S106 $\mathrm{H} 2$ and PDR, the excitation temperature indicated by the $0-0 \mathrm{~S}(11), \mathrm{S}(9)$ and $\mathrm{S}(7)$ lines is $\sim 1500-2000 \mathrm{~K}$.

\subsection{IC $1396 \mathrm{~A}$}

The $\mathrm{H}$ II region IC 1396 is illuminated by an $\mathrm{O} 6$ star, HD 206267, and has many bright-rimmed globules associated with it, one of which is denoted IC $1396 \mathrm{~A}$ and which has a somewhat cometary shape (e.g. Patel et al. 1995; Weikard et al. 1996). Two positions were observed in this globule, one situated on the bright rim facing the star, and the other 0.5 arcminute inside, denoted Pos 2 and Pos 1 respectively in Table 2. Spectra of several pure rotational $\mathrm{H}_{2}$ lines observed toward the Pos 2 position are shown in the left panel of Figure 2, whilst the resulting extinction corrected population diagram is shown in the right panel. The excitation temperature decreases with increasing depth into the globule, as shown in Table 2, and as might be expected.

\section{X-ray Dominated Regions}

X-ray dominated regions, or XDRs, arise when X-rays penetrate molecular clouds and ionise atomic and molecular material, producing fast electrons. These fast electrons then interact with the ambient gas, heating it so that thermal excitation of the rotation-vibration levels occur. The X-ray photoelectrons may also produce excited $(v, J)$ levels in a manner analogous to ultraviolet pumping. There is as yet no clear-cut case where molecular hydrogen is unambiguously heated by X-rays. This is simply because where X-rays exists, so do ultravio- 

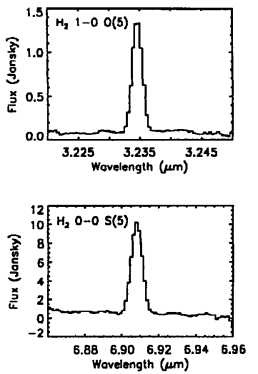
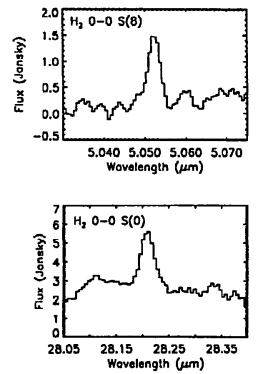

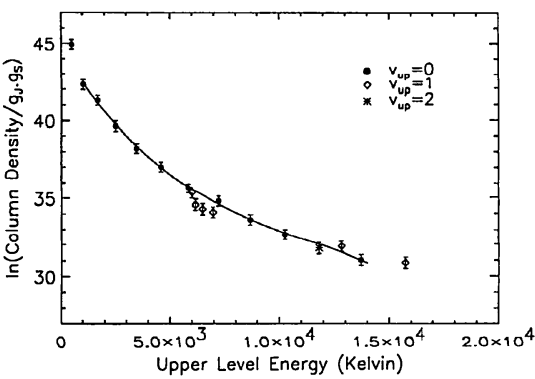

Figure 3. A selection of $\mathrm{H}_{2}$ spectra toward a position in the supernova remnant RCW 103, and the resulting extinction corrected population diagram with a cubic fit to the $v=0$ levels.

let photons and shocks (e.g. supernova remnants). However, Draine \& Woods (1990) put forward a convincing case that X-rays are responsible for most of the extremely bright 1-0 S(1) emission from the starburst galaxy NGC 6240, whilst Draine \& Woods (1991) suggest that the $\mathrm{H}_{2}$ emission from the supernova remnant RCW 103 is also produced by X-rays.

\subsection{RCW 103}

The supernova remant RCW 103 has been studied extensively in the past in the near-infrared by Oliva et al. $(1989,1990,1999)$. They showed that the remnant blast wave is interacting with the interstellar medium and producing very bright emission in lines of $\mathrm{Fe}$ II and $\mathrm{H}_{2}$. The Fe II emission coincides with the optical, radio and X-ray emission, but the $\mathrm{H}_{2}$ emission occurs 20-30 arcseconds outside (i.e. in front) of it. This poses a problem in that standard shock excitation of $\mathrm{H}_{2}$ predicts that the $\mathrm{H}_{2}$ would reside either behind or coincident with the optical emission. Extinction arguments cannot be applied since the extinction to all of the optical, $\mathrm{H}$ II and $\mathrm{H}_{2}$ emission is independently observed to be the same. Further, the $\mathrm{H}_{2}$ spectrum "looks" thermal. Therefore, X-rays have been proposed as a possible excitation mechanism. The left panel of Figure 3 shows a selection of $\mathrm{H}_{2}$ lines detected using the ISO-SWS, whilst the right panel displays the resulting extinction corrected population diagram. The excitation temperature between the $J=2$ and 3 levels is $\sim 190 \mathrm{~K}$ with a column density of order $2.4 \times 10^{21}$ $\mathrm{cm}^{-2}$, whilst that defined analogously to the shocks and PDRs above is $\sim 660 \mathrm{~K}$ and $1.6 \times 10^{20} \mathrm{~cm}^{-2}$. As found by Oliva et al. (1990) the population diagram is very reminiscent of that expected from a shock. However, the apparent deficit of emission in the 1-0 O-branch lines may be a signature of at least a non-thermal component, rather than, say, a sub-thermal population.

\subsection{SS 433}

Six knots of infrared emission were discovered toward the X-ray binary source SS 433 by Band (1987) using IRAS data. Wang et al. (1990) proposed that the knots were due to an interaction between the SS 433 jets and the interstellar medium. Spectra taken using the ISO-SWS, shown in Figure 4, demonstrate 

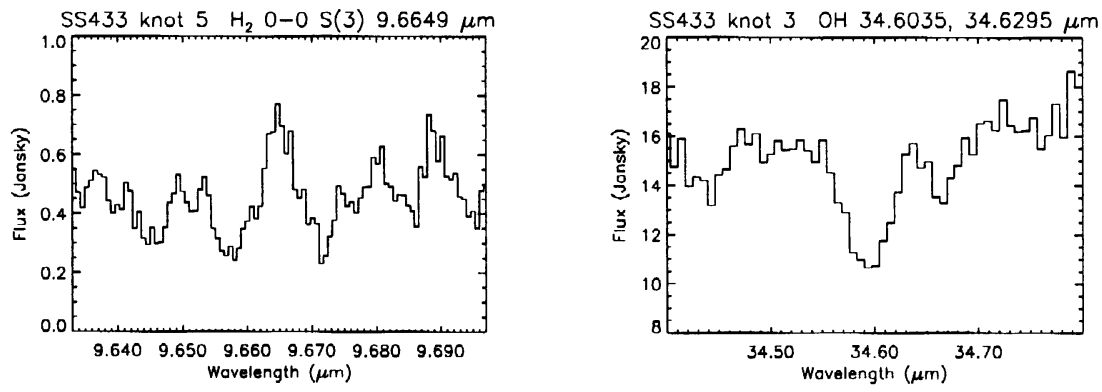

Figure 4. Spectra of the $\mathrm{H}_{2} 0-0 \mathrm{~S}(3)$ and unresolved $\mathrm{OH}$ doublet lines toward the infra-red knots 5 and 3 respectively of the X-ray binary SS 433.

that molecular material is present in the direction of the knots, but as yet do not clearly identify X-rays as being the heating agent. Band (1987) suggests that there are several unrelated molecular clouds along the line-of-sight, indicated by different velocities. Indeed the heliocentric velocity of the $\mathrm{OH}$ absorption line varies between -200 and $+100 \mathrm{~km} \mathrm{~s}^{-1}$ between knots 3 and 5 , which are only separated by $\sim 1.5$ arcminutes on the sky, whereas the $\mathrm{H}_{2} 0-0 \mathrm{~S}(3)$ line remains relatively constant at $0-30 \mathrm{~km} \mathrm{~s}^{-1}$. The $\mathrm{H}_{2}$ emission probably does not arise from the same gas as the $\mathrm{OH}$ absorption, given the implied difference in gas temperatures, and their observed velocity difference.

\section{Ortho-Para Ratio}

In equilibrium at the temperature of the warm gas component observed in shocks, PDRs and XDRs, i.e. $400-800 \mathrm{~K}$, the ortho-para ratio is expected to be equal to the ratio of their statistical weights, i.e. $3: 1$ (e.g. Burton, Hollenbach, \& Tielens 1992). In a population diagram, a non-equilibrium value of the $\mathrm{o} / \mathrm{p}$ ratio would be revealed by a separation of the ortho and para levels. For most of the sources considered in Tables 1 and 2, and those shown in Figures 1, 2 and 3, there appears no such systematic separation beyond the uncertainties and one can conclude that the $o / p$ ratio is in equilibrium at a value of 3 . Van den Ancker (1999) also finds no evidence of a non-equilibrium value of the $\mathrm{o} / \mathrm{p}$ ratio in an independent sample of both shocks and PDRs. However, there is at least one shock source so far observed, namely HH54 by Neufeld et al. (1998), where there is a systematic separation of the ortho and para levels, and although the gas temperature is $\sim 650 \mathrm{~K}$ the inferred o/p ratio is only $1.2 \pm 0.4$. They interpret this as implying that it is a legacy of an earlier stage in the thermal history of the gas, when it had reached equilibrium at a temperature of $\sim 90 \mathrm{~K}$, and suggest an upper limit on the time that the gas has been warm of $\leq 5000$ years. Their observations were made with the SWS, but have since been reproduced by Cabrit et al. (1999) using ISOCAM.

There are also cases of PDRs now appearing in the literature where a nonequilibrium value of the $\mathrm{o} / \mathrm{p}$ ratio has been inferred. For example, using the SWS Fuente et al. (1999) infer an o/p ratio of $1.5-2$ in the reflection nebula 
NGC 7023, where the gas temperature is 300-700 K. They explain their result as due to a dynamical PDR, in which cool gas (say $\leq 100 \mathrm{~K}$ for which the $\mathrm{o} / \mathrm{p}$ ratio is $<1$ ) is continually fed into the PDR as it advances into the molecular cloud. The gas is heated to $\geq 200 \mathrm{~K}$, but then leaves the PDR before it has time to attain an o/p ratio of 3 . Moutou et al. (1999), using the SWS, additionally infer an o/p ratio of $\sim 1$ toward both NGC 7023 and NGC 2023.

It is however pertinent to point out some of the inherent dangers in concluding non-equilibrium o/p ratios from ISO data. When using data from the SWS one must be careful with the $0-0 \mathrm{~S}(2)$ transition at $12.3 \mu \mathrm{m}$. This part of the SWS wavelength range suffers severe fringing effects, where the fringe period is about equal to the expected line width. In sources with only a little continuum, obtaining a reliable flux for this line can therefore be difficult. Further, the $0-0$ $\mathrm{S}(3)$ transition suffers from severe extinction effects, since it is at the peak of the silicate dust absorption band. Obtaining a reliable flux for this line is therefore also difficult, since it depends on an uncertain absolute extinction value, an uncertain extinction law and an uncertain extinction geometry (e.g. screen or mixed gas and dust). Further, if a temperature gradient exists, as might be expected, different $\mathrm{H}_{2}$ transitions will likely sample different depths into the cloud since they are most sensitive in different temperature regimes. This could be especially true for the $0-0 \mathrm{~S}(0)$ transition, for which the upper level is a factor of 2 different to the upper level of the 0-0 S(1) line, i.e. $510 \mathrm{~K}$ as opposed to $1015 \mathrm{~K}$. The $\mathrm{S}(0)$ line is therefore likely to sample gas at a significantly lower temperature than the $S(1)$ line, producing a natural separation in a population diagram (e.g. Figures 2 and 3). The larger SWS aperture used for the $S(0)$ line may only exacerbate this effect.

\section{External Galaxies}

One of the major aims behind $\mathrm{H}_{2}$ spectroscopy of Galactic sources is to extrapolate the results to extragalactic sources and thereby identify the dominant excitation mechanism, e.g. shock or PDR, as well as how much of the mass is contained within warm $\mathrm{H}_{2}$. Several studies have appeared in the literature, e.g. Kunze et al. (1996) on the colliding galaxies NGC 4038/4039, Rigopoulou et al. (1996) on the starburst NGC 3256, Sturm et al. (1996) on the ULIRG Arp 220, Valentijn et al. (1996) on the moderate starburst NGC 6946, Sturm et al. (1999) on the Seyfert NGC 4151, Kunze et al. (1999) on a sample of starbursts and Seyferts and Spoon et al. (in preparation) on the starburst NGC 4945. In all cases at least 2 of the 0-0 S(0) through $S(7)$ lines were observed, and a typical inferred excitation temperature between the $J=2$ and $J=3$ levels is $\sim 150 \mathrm{~K}$, such that the mass of warm $\mathrm{H}_{2}$ accounts for $0.2-20 \%$ of the total molecular mass determined from $\mathrm{CO}$ observations. The question of what is heating the $\mathrm{H}_{2}$, especially important when considering the ultimate energy source behind ULIRGs, i.e. starburst or AGN, is still an open issue. On the basis of Tables 1 and 2 one might infer that PDRs are in general slightly cooler than shocks. Rigopoulou et al. (private communication) find that starbursts and AGN have quite similar $\mathrm{H}_{2}$ properties, although temperatures for AGN may be a little cooler, but the statistics are still quite low. Kunze et al. (1999) infer that PDRs are heating the gas in starbursts and some Seyferts, but that a combination of 
PDRs and shocks is plausible for the Seyferts. Of course, within the ISO beam such a combination would not be unexpected.

Recently, Valentijn \& van der Werf (1999) detected large-scale $\mathrm{H}_{2}$ emission in the disk of the normal edge-on spiral galaxy NGC 891. From their $0-0 \mathrm{~S}(0)$ and $\mathrm{S}(1)$ observations, they inferred the presence of two temperature components, one at 150-230 K scattered throughout the disk, and another at 80-90 K which dominates in the outer regions. Interestingly, for an ortho-para ratio of 2-3 the mass they calculate in the cool component outweighs the $\mathrm{H}$ I by a factor of 5-15, in which case the missing matter problem of spiral galaxies may be resolved. However, for an ortho-para ratio closer to 1 , the inferred $\mathrm{H}_{2}$ mass is lower.

\section{Deuterium Abundance}

Wright et al. (1999) and Bertoldi et al. (1999b) observed rotational lines of HD toward the Orion Bar and Orion shock $\mathrm{Pk} 1$ respectively. These were the first ever detections of $\mathrm{HD}$ rotational emission lines outside of the solar system. In the former case the $112 \mu \mathrm{m} J=1-0$ line was observed, which resulted in an inferred $[\mathrm{D} / \mathrm{H}]$ of $(1.0 \pm 0.3) \times 10^{-5}$, whilst in the latter the $19.4 \mu \mathrm{m} J=6-5$ transition was detected, with an implied $[\mathrm{D} / \mathrm{H}]$ of $(0.76 \pm 0.29) \times 10^{-5}$.

\section{Conclusions}

Using the spectroscopic instruments on board the Infrared Space Observatory, the $\sim 2000 \mathrm{~K}$ and non-thermal components of $\mathrm{H}_{2}$, known from ground-based telescopes, are still observed toward Galactic shocks and PDRs respectively, but are seen to form only a part of a distribution of temperatures. For instance, observations using the SWS of the $0-0 \mathrm{~S}(1)$ through $\mathrm{S}(5)$ lines ubiquitously reveal a component at about $600-800 \mathrm{~K}$ in shocks, and about $400-700 \mathrm{~K}$ in PDRs, whilst the lowest rotational transition at $28.2188 \mu \mathrm{m}$ reveals a component at $100-200 \mathrm{~K}$. This warm gas contains significantly more mass than the hot (2000 $\mathrm{K})$ component, and one can now say that with ISO the bulk of the gas in these sources is being probed. Higher temperature gas is also seen, especially toward shocks. Further, imaging observations with ISOCAM have been obtained in several of the pure rotational transitions, thereby allowing a spatial study of the excitation, whilst the deuterium abundance has been determined from combined SWS and LWS observations.

Although with the depletion of its liquid helium supply in April 1998 ISO is no longer operational, the work in reducing, analysing and interpreting the wealth of $\mathrm{H}_{2}$ data will go on for some time. This paper presents only a first attempt at putting that data into some sort of scientific and statistical perspective, and it is expected that by the time of the next IAU Astrochemistry symposium much progress toward addressing the issues outlined in the introduction will have been made. It is equally certain however that even more questions will be raised.

Acknowledgments. I am extremely grateful to my $\mathrm{H}_{2}$ and $\mathrm{HD}$ collaborators Ralf Timmermann, Siegfried Drapatz, Frank Bertoldi, Dirk Rosenthal, Paul van der Werf, Ewine van Dishoeck, Annemieke Boonman, Wing-Fai Thi, 
Gerd-Jan van Zadelhoff, Pierre Cox, Tino Oliva, Alan Moorwood and Dimitra Rigopoulou, from all of whom I have learnt a little something.

\section{References}

Band, D.L. 1987, PASP, 99, 1269

Beck, S.C., Lacy, J.H., \& Geballe, T.R. 1979, ApJ, 234, L213

Beckwith, S., Persson, S.E., Neugebauer, G., \& Becklin, E.E. 1978, ApJ, 223, 464

Bertoldi, F., Draine, B.T., Drapatz, S., et al. 1999a, in The Physics and Chemistry of the Interstellar Medium, $3^{r d}$ Cologne-Zermatt Symposium, eds. V. Ossenkopf et al., GCA-Verlag Herdecke, 120

Bertoldi, F., Timmermann, R., Rosenthal, D., Drapatz, S., \& Wright, C.M. 1999b, A\&A, 346, 267

Burton, M.G., Hollenbach, D.J., \& Tielens, A.G.G.M. 1992, ApJ, 399, 563

Burton, M.G. \& Haas, M.R. 1997, A\&A, 327, 309

Cabrit, S., Bontemps, S., Lagage, P.O., et al. 1999, in The Universe as seen by ISO, eds. P. Cox \& M.F. Kessler, ESA SP-427, Noordwijk, 449

Cesarsky, D., Cox, P., Pineau des Forêts, G., et al. 1999, A\&A, 348, 945

Draine, B.T. 2000, to be published in $\mathrm{H}_{2}$ in Space, eds. F. Combes \& G. Pineau des Forêts, Cambridge University Press

Draine, B.T. \& McKee, C.F. 1993, ARA\&A, 31, 373

Draine, B.T. \& Bertoldi, F. 1999, in The Universe as seen by ISO, eds. P. Cox \& M.F. Kessler, ESA SP-427, Noordwijk, 553

Draine, B.T. \& Woods, D.T. 1990, ApJ, 363, 464 1991, ApJ, 383, 621

Flower, D.R. \& Pineau des Forêts, G. 1999, MNRAS, 308, 271

Fuente, A., Martin-Pintado, J., Rodriguez-Fernandez, N.J., et al. 1999, ApJ, $518, \mathrm{~L} 45$

Gautier, T.N., Fink, U., Treffers, R.R., \& Larson, H.P. 1976, ApJ, 207, L129

Genzel, R. \& Stutzki, J. 1989, ARA\&A, 27, 41

Goldsmith, P.F. \& Langer, W.D. 1999, ApJ, 517, 209

Gredel, R. 1994, A\&A, 292, 580

Hollenbach, D.J. \& Salpeter, E.E. 1971, ApJ, 163, 155

Hollenbach, D.J. \& Tielens, A.G.G.M. 1997, ARA\&A, 35, 179

Kaufman, M.J. \& Neufeld, D.A. 1996, ApJ, 456, 611

Kessler, M.F., Steinz, J.A., Anderegg, M.E., et al. 1996, A\&A, 315, L27

Knacke, R.F. \& Young, E.T. 1981, 249, ApJ, L65

Kunze, D., Rigopoulou, D., Lutz, D., et al. 1996, A\&A, 315, L101

Kunze, D., Rigopoulou, D., Genzel, R., \& Lutz, D. 1999, in The Universe as seen by ISO, eds. P. Cox \& M.F. Kessler, ESA SP-427, Noordwijk, 909

Moutou, C., Verstraete, L., Sellgren, K., \& Leger, A. 1999, in The Universe as seen by ISO, eds. P. Cox \& M.F. Kessler, ESA SP-427, Noordwijk, 727

Neufeld, D.A., Melnick, G.J., \& Harwit, M. 1998, ApJ, 506, L75

Oliva, E. \& Moorwood, A.F.M. 1988, A\&A197, 261

Oliva, E., Moorwood, A.F.M., \& Danziger, I.J. 1989, A\&A, 214, 307

1990, A\&A, 240, 453

Oliva, E., Moorwood, A.F.M., Drapatz, S., Lutz, D., \& Sturm, E. 1999, A\&A, 343,943 
Parmar, P.S., Lacy, J.H., \& Achtermann, J.M. 1991, ApJ, 372, L25

1994, ApJ, 430, 786

Patel, N.A., Goldsmith, P.F., Snell, R.L., Hezel, T., \& Xie, T. 1995, ApJ, 447, 721

Richter, M.J., Graham, J.R., Wright, G.S., Kelly, D.M., \& Lacy, J.H. 1995, ApJ, 449, L83

Rigopoulou, D., Lutz, D., Genzel, R., et al. 1996, A\&A, 315, L125

Rosenthal, D., Bertoldi, F., Drapatz, S., \& Timmermann, R. 1999, in The Universe as seen by ISO, eds. P. Cox \& M.F. Kessler, ESA SP-427, Noordwijk, 561

Shull, J.M. \& Beckwith, S. 1982, ARA\&A, 20, 163

Sturm, E., Lutz, D., Genzel, R., et al. 1996, A\&A, 315, L133

Sturm, E., Alexander, T., Lutz, D., et al. 1999, ApJ, 512, 197

Thi, W.F., van Dishoeck, E.F., Black, J.H., et al. 1999, in The Universe as seen by ISO, eds. P. Cox \& M.F. Kessler, ESA SP-427, Noordwijk, 767

Timmermann, R., Bertoldi, F., Wright, C.M., et al. 1996, A\&A, 315, L281

Treffers, R.R., Fink, U., Larson, H.P., \& Gautier, T.N. 1976, ApJ, 209, 793

Valentijn, E.A., van der Werf, P.P., de Graauw, Th., \& de Jong, T. 1996, A\&A, $315, \mathrm{~L} 145$

Valentijn, E.A. \& van der Werf, P.P. 1999, ApJ, 522, L29

van den Ancker, M.E. 1999, $\mathrm{PhD}$ thesis, University of Amsterdam

van den Ancker, M.E., Wesselius, P.R., Tielens, A.G.G.M., \& Waters, L.B.F.M. 1998, in ISO's view on Stellar Evolution, eds. L.B.F.M. Waters et al., Ap\&SS, 255,69

van den Ancker, M.E., Wesselius, P.R., Tielens, A.G.G.M., van Dishoeck, E.F., \& Spinoglio, L. 1999, A\&A, 348, 877

van Dishoeck, E.F., Wright, C.M., Cernicharo, J., et al. 1998, ApJ, 502, L173

Wang, Z.-R., McCray, R., Chen, Y., \& Qu, Q.-Y. 1990, A\&A, 240, 98

Weikard, H., Wouterloot, J.G.A., Castets, A., Winnewisser, G., \& Sugitani, K. 1996, A\&A, 309, 581

Weingartner, J.C. \& Draine, B.T. 1999, in The Universe as seen by ISO, eds. P. Cox \& M.F. Kessler, ESA SP-427, Noordwijk, 783

Wright, C.M., Drapatz, S., Timmermann, R., et al. 1996, A\&A, 315, L301

Wright, C.M., Timmermann, R., \& Drapatz, S. 1997, in First ISO Workshop on Analytical Spectroscopy, eds. A.M. Heras et al., ESA SP-419, Noordwijk, 311

Wright, C.M., van Dishoeck, E.F., Cox, P., Sidher, S., \& Kessler, M.F. 1999, ApJ, 515, L29 


\section{Discussion}

A. Richards: Please show the location of the detection of $\mathrm{H}_{2}$ in SS 433; the detection of molecular material in an X-ray binary is exciting.

C.M. Wright: The detections of the $\mathrm{H}_{2} 0-0 \mathrm{~S}(3)$ line were made towards the infrared knots 3 and 5 found by Band (1987, PASP, 99, 1269). But more work is needed to confirm that the lines do not arise from somewhere else along the line of sight, e.g. from the W50 radio source.

S. Pak: You mentioned that we can measure the dark mass in NGC 891. But the excitation temperature of the $\mathrm{H}_{2}$ rotational line is over several $100 \mathrm{~K}$. How can we trace the cold gas with the rotational line?

C.M. Wright: The authors, Valentijn \& van der Werf, of the paper of which you speak infer two temperature components, one at $150-230 \mathrm{~K}$, and another at 80-90 K, from the $\mathrm{H}_{2} 0-0 \mathrm{~S}(0)$ and $\mathrm{S}(1)$ transitions. It is the colder component which contains the bulk of the mass.

J.H. Black: You mentioned that the $\mathrm{T}_{e x}$ for rotational transitions of $J_{u p}=3-7$ are not explained by pre-ISO PDR models. Are they explained by "post-ISO PDR models" and if so, what is the basic difference between them and the "pre-ISO models" ?

C.M. Wright: Whilst at least 2 of the relevant lines, the $\mathrm{S}(1)$ and $\mathrm{S}(2)$, were observed pre-ISO in 1 object, the Orion Bar by Parmar et al. in the early 90 's, only ISO has allowed the whole suite of lines to be measured ubiquitously in many sources. So before ISO the PDR models could not reliably be tested. The relevant lines simply weren't observed. As far as I know, there are no new "post-ISO" PDR models self-consistently producing the higher than expected excitation temperatures. 